Nephrologe 2021 · 16:380-385 https://doi.org/10.1007/s11560-021-00536-9 Angenommen: 1. September 2021 Online publiziert: 24. September 2021 ๑) Springer Medizin Verlag GmbH, ein Teil von Springer Nature 2021

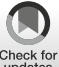

\section{Curriculum „Palliative nephrologische Betreuung“}

\author{
Christoph Gerhard' . Wolfgang Pommer ${ }^{2}$ \\ ${ }^{1}$ Niederrheinische Akademie für medizinische Fort- und Weiterbildung, Dinslaken, Deutschland \\ ${ }^{2}$ Kuratorium für Dialyse und Nierentransplantation $(\mathrm{KfH})$ - Bildungszentrum, Neu-Isenburg, Deutschland
}

\title{
Zusammenfassung
}

Palliativmedizinische Aspekte wie Dialysevorenthalt und -abbruch, Einleitung einer konservativen Therapie sowie kooperative Betreuung am Lebensende sind zunehmend Teil der nephrologischen Regelversorgung geworden. Die entsprechende Wissensvermittlung palliativmedizinischer Grundsätze fehlt bislang in Aus- und Weiterbildung. In diesem Konsensuspapier wird für das nephrologische Team eine strukturierte kurrikuläre Weiterbildung zu den Grundprinzipien einer palliativen Betreuung vorgeschlagen.

\section{Schlüsselwörter}

Nephrologie · Terminale Betreuung · Palliativmedizin · Fortbildung $\cdot$ Konsensus

Demographische Entwicklung und altersassoziierte Komorbidität haben in Europa, in den USA, Kanada und in Australien zu einer Zunahme älterer und hochaltriger Menschen in den nephrologischen Behandlungsprogrammen geführt. Dabei wird zunehmend die Begrenztheit der Dialyseoptionen im hohen Lebensalter in Bezug auf Lebensverlängerung und Lebensqualität realisiert $[9,17,22,25$, 26]. Die Einjahresmortalität älterer Dialysepatienten (> 75 Jahre) beträgt 20-45\% $[9,17,26]$. Ärztliche Aufgabe im Stadium des fortgeschrittenen oder terminalen Nierenversagens (ESRD) ist die Abwägung eines konservativen Vorgehens oder des Angebots einer Nierenersatztherapie mit dem Ziel, Patienten, Zugehörige und Betreuende entsprechend $z u$ beraten. Im Verlauf der Dialysebehandlung und Nierentransplantation kann bei progredienter Komorbidität oder lebensbeeinträchtigenden Akutkomplikationen wie in der aktuellen COVID-19-Pandemie [7] erneut eine Abwägung der Sinnhaftigkeit einer fortgeführten Nierenersatztherapie notwendig werden [15, 20]. Im Ergebnis leitet sich hieraus die Erkenntnis einer nur sehr begrenzten Behandlungsprognose für die Betroffenen her. Der Kreis der Betroffenen umfasst in diesem Sinne nicht nur den Patienten und sein persönliches und fami- liäres Umfeld, sondern auch das Team der Behandler selbst: Pflegende und Ärzte.

\section{Curriculäre Ausbildung}

Die Betreuung am Lebensende beinhaltet Kenntnisse und Kompetenzen, die (bislang) nicht in der fachspezifischen nephrologischen Weiterbildung erworben werden. Die Aufmerksamkeit gegenüber den besonderen Problemen in der Versorgung nierenkranker Menschen am Lebensende ist sowohl in der Erwachsenen- wie in der Kindernephrologie $[19,27]$ hoch. Eine Vielzahl von strukturellen und motivationalen Hürden behindert jedoch die Umsetzung palliativmedizinischer Angebote, die zu einer Entlastung in dieser Betreuungssituation und zu einer Befriedigung von Wünschen und Bedürfnissen der Betroffenen am Lebensende beitragen könnte.

\section{॥ Das Curriculum beinhaltet ein Angebot zum Kenntniserwerb einer strukturierten Betreuung am Lebensende}

Das Curriculum „Palliative nephrologische Betreuung" beinhaltet ein Angebot an Ärztinnen und Ärzte sowie Pflegende des Fachgebiets zum Kenntniserwerb einer strukturierten Betreuung am Lebensende 


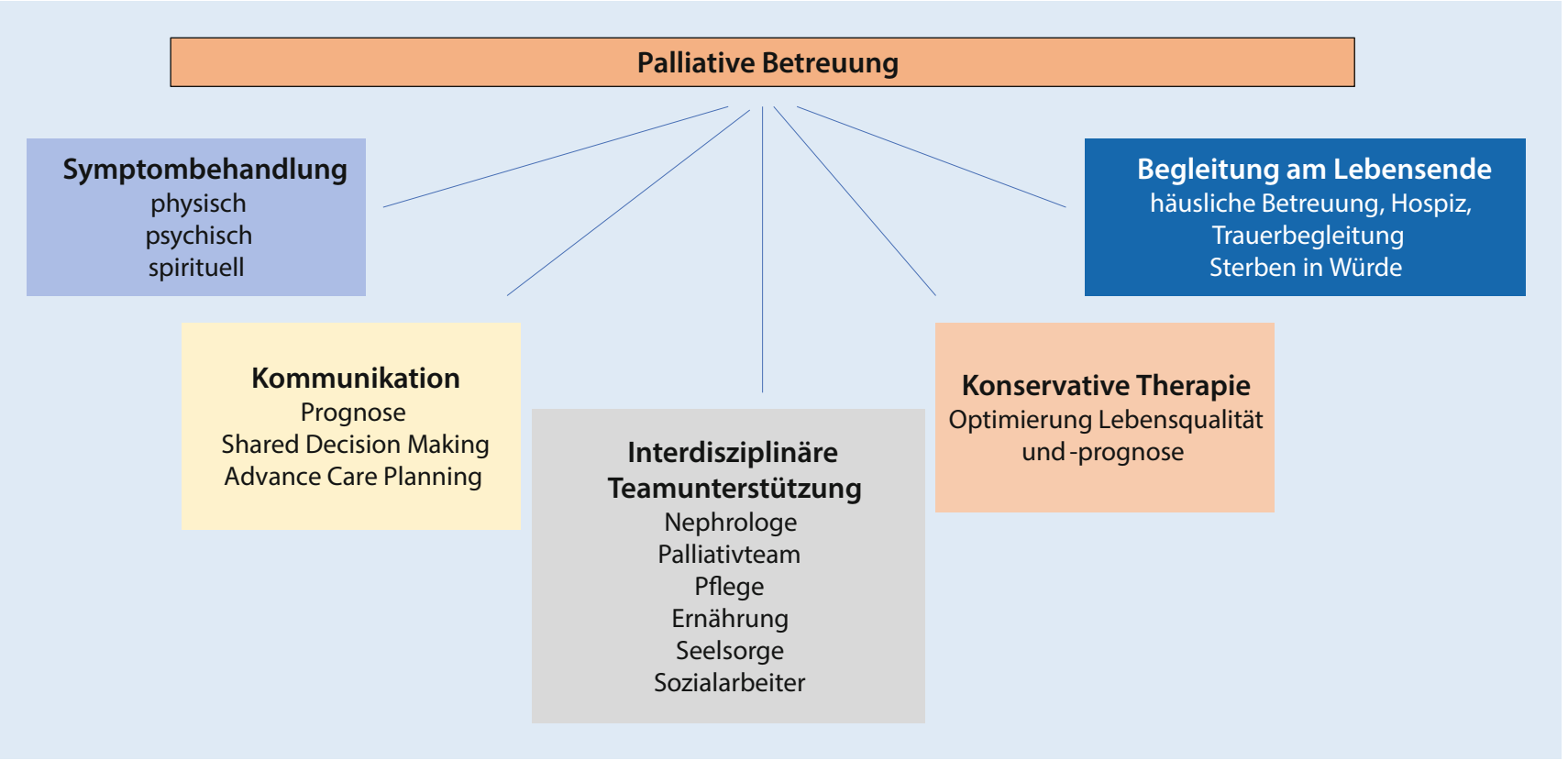

Abb. $1 \Delta$ Aspekte einer palliativen nephrologischen Betreuungsstruktur. (Modifiziert nach [4])

(- Abb. 1). Es orientiert sich an Erfahrungen aus dem englischsprachigen Raum [30], die bereits in Form eines curriculären Weiterbildungsangebots $[5,6]$ umgesetzt wurden.

\section{Fortbildungsinhalte}

Inhaltlich wird in insgesamt 16 Unterrichtseinheiten (UE) mit einem zusätzlichen zeitsouveränen Literaturstudium von 4 UE eine Einführung in die palliativmedizinischen Grundlagen nichttumorerkrankter Patienten angeboten (- Tab. 1). Weitere Abschnitte beinhalten die nichtrenalen Begleiterkrankungen und die Symptomlast bei fortgeschrittener Niereninsuffizienz, das dazu notwendige Assessment sowie die Anwendung von Prognosescores. Symptombehandlung und die Besonderheiten der medikamentösen Therapie bei eingeschränkten Nierenfunktion sind ein weiterer Schwerpunkt. Im Kontext der unterschiedlichen Optionen einer Nierenersatztherapie werden die Aspekte konservative Therapie, Angemessenheit unterschiedlicher Dialyseformen sowie Dialyseabbruch und -vorenthalt hinsichtlich der Lebensqualität und ethischer Grundsätze diskutiert. Ein weiterer Schwerpunkt ist die gesundheitliche Vorausplanung (Advance Care Planning, APC). Die Modelle der ärztlichen Kommunikation in der Überbringung schlechter Nachrichten und die notwendigen Rahmenbedingungen dafür werden vorgestellt und im Rollenspiel geübt. Schließlich werden die möglichen Szenarien in der Sterbebegleitung am Lebensende unter Einbeziehung von Palliativstrukturen thematisiert, und der Aspekt der Bedürfnisse von An- und Zugehörigen wird reflektiert. Die Zeitachse in Verlauf und Strukturierung des ACP-Prozesses soll hier eine Orientierung geben (• Abb. 2).

\section{Zielgruppe, Lernformate und Lernziele}

Es wird postuliert, dass alle Mitglieder des Behandlungsteams in ihrer jeweils unterschiedlichen Profession (Ärzte, Pflegende, Sozialarbeiter) am Prozess der Betreuung am Lebensende („end-of-life care“) beteiligt sind. Ausdrücklich wird dies nicht nur unter dem Aspekt des Therapieprozesses selbst, sondern vielmehr auch unter dem Gesichtspunkt persönlicher Betroffenheit, subjektiver und objektiver Belastung, konfliktärer Teaminteraktion und Verfügbarkeit von strukturellen Ressourcen begriffen. Ziel ist es, neben dem Kenntniserwerb der palliativmedizinischen Handlungsoptionen (-Tab. 1) einen Gestaltungsrahmen für die Teaminteraktion und eine Hal- tung der Aufmerksamkeit und bewussten Wahrnehmung von Teamproblemen und Möglichkeiten einer befriedigenden Konfliktlösung zu erarbeiten. Daraus resultiert die prinzipielle Notwendigkeit eines Präsenzformats in einer überschaubaren Gruppengröße.

Die Teilnehmer und Teilnehmerinnen sollen befähigt werden,

- die erworbenen palliativmedizinischen Kenntnisse in die nephrologische Therapieplanung zu integrieren;

- insbesondere die spezielle Symptombelastung am Lebensende zu ermitteln und angemessen zu therapieren;

- sich in die Rolle der Betroffenen einzufühlen und deren Wünsche und Bedürfnisse in die Behandlungspraxis einzubeziehen;

- eine angemessene Kommunikation zu führen und einen dafür notwendigen Handlungsrahmen zu realisieren.

Als Lehr- und Lernformate hierfür sind geeignet:

- Wissenstransfer mit interaktiv-reflektierenden Elementen;

- Kleingruppenarbeiten, Fallpräsentationen, Feedbackrunden;

- Rollenspiel, Selbsterfahrungsübungen. 
Tab. 1 Curriculum „Palliative nephrologische Betreuung"

UE Inhalt

2 Palliative Grundlagen

Modelle von Krankheitsverläufen

Palliativ-Care-Konzepte (Total-Pain-

Modell, bedürfnisorientierte Sichtweise)

Radikale Patientenorientierung

Parallelität von kurativem und palliativem Handeln

\begin{tabular}{|l|l|}
\cline { 2 - 2 } & Versorgungskonzepte und -strukturen \\
\cline { 2 - 3 } & Autonomie und Lebensqualität \\
\hline 2 & $\begin{array}{l}\text { Komorbidität und Prognose bei Nie- } \\
\text { renerkrankungen }\end{array}$ \\
\cline { 2 - 2 } & $\begin{array}{l}\text { Typische Komorbidität (Angst, Depres- } \\
\text { sion, Schmerz u. ä.) }\end{array}$ \\
\cline { 2 - 2 } & $\begin{array}{l}\text { Total-Pain-Konzept (Woran leidet der } \\
\text { Patient?) }\end{array}$ \\
\hline
\end{tabular}

Prognosescores und Überlebenswahrscheinlichkeit

2 Symptomerfassung und -therapie Assessment

Schmerz- und Symptommanagement Kognitive Veränderungen, Delir

2 Besonderheiten der Schmerz- und Symptombehandlung

Substanzauswahl, Dosierung Pflegerische Maßnahmen

2 Nierenersatztherapie und Lebensqualität

Konservative Behandlung

Lebensqualität unter Dialyseverfahren

Dialyseabbruch und -vorenthalt

2 Ethische Fragen

Grundlegende Prinzipien

Entscheidungskonflikte

Gesetzliche Vorgaben

2 Advance Care Planning

Vorausplanung, Einwilligungsfähigkeit

Differenzierte Entscheidungsfindung

Gesprächsführung, -setting

2 Sterben mit fortgeschrittener Nierenerkrankung

Sterbeverläufe

Transkulturelle Besonderheiten

Wo sterben die Betroffenen?

4 Selbststudium

UE Unterrichtseinheit (45 min)
Lernziele umfassen das Verständnis dafür,

- dass palliativmedizinische Angebote zu einer höheren Wunsch- und Bedürfnisbefriedigung von Menschen am Lebensende beitragen;

- dass palliativmedizinische Angebote rechtzeitig in das nephrologische Behandlungskonzept einbezogen werden sollten und dies zu einer Symptomentlastung und höheren Behandlungsqualität am Lebensende führt;

- dass ein palliativmedizinisches Konzept Teil der gesundheitlichen Vorausplanung ist und zu einer wesentlichen Entlastung des Betreuungsteams, der betroffenen Menschen und ihrer Zugehörigen beiträgt.

\section{Begründung und aktueller Wissenstand}

Aus dem überproportionalen Zuwachs Älterer und hochaltriger Patienten in den nephrologischen Behandlungsprogrammen der Länder mit hohem Einkommen ergeben sich Behandlungsbesonderheiten, die im Sinne einer "geriatrischen Nephrologie" zusätzliche Weiterbildungsaspekte erfordern. Diese sind in einem Onlinecurriculum der American Society of Nephrology thematisch aufbereitet [16]. 2020 wurde das Kidney Supportive Care Core Curriculum der National Kidney Foundation initiiert [4]. Das Konzept einer nephrologischen Palliativbetreuung ist seit fast 15 Jahren in einem Praxishandbuch formuliert [29] und dient als Inhaltsgerüst eines speziellen Curriculums der nephropalliativen Betreuung. Die Integration eines palliativmedizinischen Ansatzes in die Nephrologie ist durch die Ähnlichkeit der Symptombelastung und Überlebensprognose von Krebserkrankten mit anderen chronisch Kranken - darunter Nierenkranke gerechtfertigt [14, 24, 28]. Eine palliative Betreuung lebt von Interdisziplinarität ihrer Akteure $[8,23]$ sowie im Sinne einer Nachhaltigkeit von einem Modell einer allgemeinen und spezialisierten Versorgung [21]. Unter der Fragestellung: „Sollte die Rolle der Palliativbetreuung bei ESRD ausgeweitet werden", formulieren Kurella Tamura et al. [10, 11] einen 5-Punkte-Plan, um Hürden zum Zugang von Patienten mit ESRD zur Palliativversorgung zu überwin- den. Die Einstellungen der Nephrologen in Europa zur Problematik der palliativen Betreuung ist nach einem EDTA(European Dialysis and Transplant Association)-Survey heterogen $[2,12]$; dieses Resultat wird durch die Ergebnisse einer jüngeren Umfrage in Deutschland unterstrichen [19]. So werden die Probleme der Betreuung Nierenkranker in der Endphase durchaus realisiert, eine strukturierte palliative Betreuungssituation ist aber nur in $20 \%$ der Zentren etabliert [17]. Als Hürden wurden dabei fehlende Ressourcen, aber auch mangelnde Kenntnisse in den Grundlagen und der Umsetzung eines palliativen Konzepts formuliert [17, 19]. Ähnliche Ergebnisse finden sich in einem weltweiten Survey, gemäß dem konservative Behandlungsmaßnahmen verfügbar sind, aber wesentliche qualitative Aspekte wie ein multidisziplinärer Teamansatz, eine gemeinsame Entscheidungsfindung (Shared Decision Making) oder die Berücksichtigung kultureller, spiritueller und psychologischer Unterstützung zum großen Teil fehlen [13]. Das aktuelle Spektrum der palliativen nephrologischen Betreuungspraxis in der nephrologischen Pädiatrie und Erwachsennephrologie wurde kürzlich in einem Sammelband publiziert [18].

\section{॥ Eine strukturierte palliative}

Betreuungssituation ist nur in $20 \%$ der Zentren etabliert

Die Verantwortung des Arztes in der Wahrnehmung und Unterstützung der Wünsche und Bedürfnisse am Lebensende und die daraus folgende Notwendigkeit seiner (kontinuierlichen) Weiterbildung sind wiederholt thematisiert (vgl. dazu [1, 3]). Wesentliche Inhalte sind der Umgang mit konfliktären Entscheidungssituationen, mit der Ungewissheit in Bezug auf das Ergebnis einer Nierenersatztherapie im Vergleich zur konservativen Behandlung sowie die Relevanz von Prognosekriterien im Einzelfall. Gleichzeitig sollte aber eine Haltung vermittelt werden, dass der Einbezug der Palliativmedizin eine Entlastung für das nephrologische Team darstellt, von der die Betroffenen und Zugehörigen wie auch die Teammitglieder selbst profitieren $[1,29,30]$. 
Hier steht eine Anzeige.

黑 Springer 


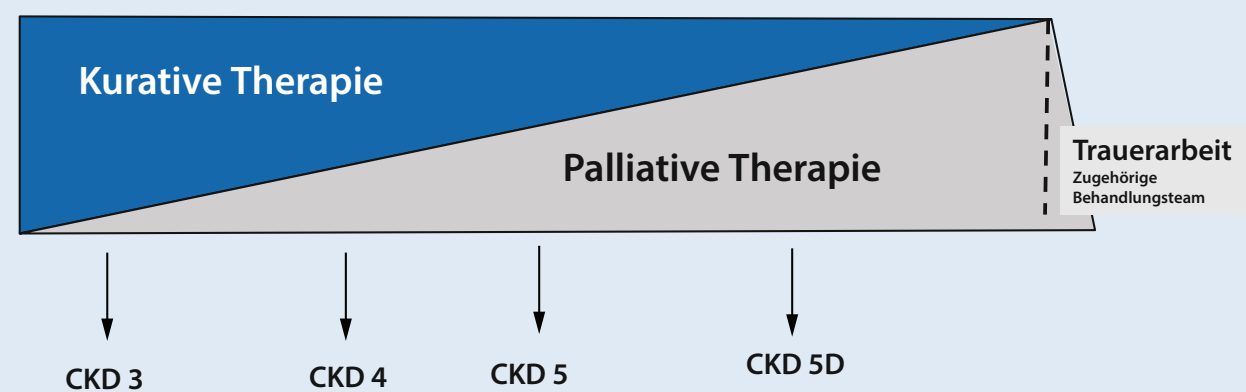

Vermeidung zusätzlicher Nierenschädigung

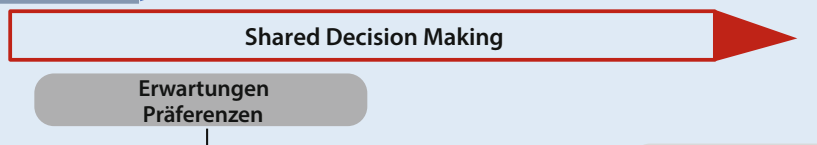

Information

Zielbestimmung

Hoffnung, Befürchtungen klären
Ziel erreicht?

Abwägung Vor-/Nachteile Therapiezieländerung
Abb. $2 \triangleleft$ Prozess der Transition von kurativer zur palliativen Therapie und vorausschauende Gesundheitsplanung (Advance Care Planning). (Modifiziert nach [18])

\section{Fazit für die Praxis}

- Die dargestellten Bedingungen der aktuellen nephrologischen Therapie verlangen auch eine Fokussierung auf die Betreuung am Lebensende, wenn aktive Therapiemaßnahmen versagen, diese aus Sicht des Patienten nicht gewünscht oder nicht erfolgversprechend sind.

- Bei einer (mutmaßlich) verbleibenden Überlebenszeit von weniger als ein Jahr sollten palliativmedizinische Versorgungsangebote realisiert werden.

- Das hier dargestellte Curriculum soll ein Fortbildungsangebot für den Erwerb von Grundkenntnissen einer verbesserten Versorgungspraxis nephrologischer $\mathrm{Pa}$ tienten am Lebensende sein.

\section{Korrespondenzadresse}

Prof. Dr. med. Wolfgang Pommer

Kuratorium für Dialyse und Nierentransplantation $(\mathrm{KfH})$ - Bildungszentrum

Martin-Behaim-Str. 20, 63263 Neu-Isenburg,

Deutschland

wolfgang.pommer@kfh.de

\section{Einhaltung ethischer Richtlinien}

Interessenkonflikt. C. Gerhard und W. Pommer geben an, dass kein Interessenkonflikt besteht.

Für diesen Beitrag wurden von den Autoren keine Studien an Menschen oder Tieren durchgeführt. Für die aufgeführten Studien gelten die jeweils dort angegebenen ethischen Richtlinien.

\section{Literatur}

1. Welsch K, Gottschling S (2021) Wishes and needs at the end of life: communication strategies, counseling, and administrative aspects. https://www.aerzteblatt.de/int/archive/article? id=218848. Zugegriffen: 30. Juli 2021

2. van Biesen W, van de Luijtgaarden MWM, Brown EA et al (2015) Nephrologists' perceptions regarding dialysis withdrawal and palliative care in Europe: lessons from a European renal best practice survey. Nephrol Dial Transplant 30:1951-1958. https:// doi.org/10.1093/ndt/gfv284

3. Blinderman CD, Billings JA (2015) Comfort care for patients dying in the hospital. $\mathrm{N}$ Engl J Med 373:2549-2561. https://doi.org/10.1056/ NEJMra1411746

4. Gelfand SL, Scherer JS, Koncicki HM (2020) Kidney supportive care: core curriculum 2020. Am J Kidney Dis 75:793-806. https://doi.org/10.1053/j.ajkd. 2019.10.016

5. Gerhard C (2013) Ausbildungsmodule - Curriculum Palliative Care für Nichttumorpatienten? Z Palliativmed 14:249-250. https://doi.org/10. 1055/s-0033-1361997

6. Gerhard C (2021) Advance care planning: Mehr Patientenautonomie am Lebensende. NierenHochdruckkrankheiten 50:237-242. https://doi. org/10.5414/NHX02193

7. Jager KJ, Kramer A, Chesnaye NC et al (2020) Results from the ERA-EDTA registry indicate a high mortality due to COVID-19 in dialysis patients and kidney transplant recipients across Europe. Kidney Int 98:1540-1548. https://doi.org/10.1016/j.kint. 2020.09.006

8. Kelley AS, Morrison RS (2015) Palliative care for the seriouslyill. NEngl JMed 373:747-755. https://doi. org/10.1056/NEJMra1404684

9. Kurella M, Covinsky KE, Collins AJ, Chertow GM (2007) Octogenarians and nonagenarians starting dialysis in the United States. Ann Intern Med 146:177-183. https://doi.org/10.7326/00034819-146-3-200702060-00006

10. Kurella Tamura M, Cohen LM (2010) Should there be an expanded role for palliative care in end-stage renal disease? Curr Opin Nephrol
Hypertens 19:556-560. https://doi.org/10.1097/ MNH.0b013e32833d67bc

11. Kurella Tamura M, Meier DE (2013) Five policies to promote palliative care for patients with ESRD. Clin J Am Soc Nephrol 8:1783-1790. https://doi.org/ 10.2215/CJN.02180213

12. van de Luijtgaarden MWM, Noordzij $M$, van Biesen W et al (2013) Conservative care in Europe-nephrologists' experience with the decision not to start renal replacement therapy. Nephrol Dial Transplant 28:2604-2612. https:// doi.org/10.1093/ndt/gft287

13. Lunney M, Bello AK, Levin A et al (2020) Availability, accessibility, and quality of conservative kidney management worldwide. Clin J Am Soc Nephrol 16:79-87.https://doi.org/10.2215/CJN.09070620

14. Moens K, Higginson IJ, Harding R, EURO IMPACT (2014) Are there differences in the prevalence of palliative care-related problems in people living with advanced cancer and eight non-cance conditions? A systematic review. J Pain Symptom Manage 48:660-677. https://doi.org/10.1016/j. jpainsymman.2013.11.009

15. Murphy E, Germain MJ, Cairns H et al (2014) International variation in classification of dialysis withdrawal: a systematic review. Nephrol Dial Transplant 29:625-635. https://doi.org/10.1093/ ndt/gft458

16. Nesbitt H, American Society of Nephrology (2021) Online curricula-geriatric nephrology. https://www.asn-online.org/education/ distancelearning/curricula/geriatrics/. Zugegriffen: 23. Apr. 2021

17. Pommer W (2021) Integrated renal-palliative care in Germany-results from a survey on end of life care in a non-profit provider. Z Gerontol Geriatr. https://doi.org/10.1007/s00391-021-01946-z

18. Pommer W, Thumfart J (2021) Palliative Nephrologie - Aktuelle Beiträge aus der Praxis. DustrieVerlag,

19. Pommer W, Wagner S, Thumfart J (2019) Conservative care, dialysis withdrawal, and palliative care: results from a survey of a non-profit dialysis provider in Germany. Kidney Blood Press Res 44:158-169. https://doi.org/10.1159/000498994 
20. Qazi HA, Chen H, Zhu M (2018) Factors influencing dialysiswithdrawal:ascoping review. BMCNephrol 19:96. https://doi.org/10.1186/s12882-018-08945

21. Quill TE, Abernethy AP (2013) Generalist plus specialist palliative care-creating a more sustainable model. N Engl J Med 368:1173-1175. https://doi. org/10.1056/NEJMp1215620

22. Rosansky SJ, Schell J, Shega J et al (2017) Treatment decisions for older adults with advanced chronic kidney disease. BMC Nephrol 18:200. https://doi. org/10.1186/s12882-017-0617-3

23. Siouta N, Van BeekK, van der Eerden ME et al (2016) Integrated palliative care in Europe: a qualitative systematic literature review of empirically-tested models in cancer and chronic disease. BMC Palliat Care 15:56. https://doi.org/10.1186/s12904-0160130-7

24. Solano JP, Gomes B, Higginson IJ (2006) A comparison of symptom prevalence in far advanced cancer, AIDS, heart disease, chronic obstructive pulmonary disease and renal disease. J Pain Symptom Manage 31:58-69. https://doi. org/10.1016/j.jpainsymman.2005.06.007

25. Steinman TI (2012) The older patient with endstage renal disease: is chronic dialysis the best option? Semin Dial 25:602-605. https://doi.org/ 10.1111/sdi.12035

26. Swidler MA (2012) Geriatric renal palliative care. JGerontol A Biol Sci Med Sci 67:1400-1409. https:// doi.org/10.1093/gerona/gls202

27. Thumfart J, Reindl T, Rheinlaender C, Müller D (2018) Supportive palliative care should be integrated into routine care for paediatric patients with life-limiting kidney disease. Acta Paediatr 107:403-407.https://doi.org/10.1111/apa.14182

28. Wachterman MW, Pilver C, Smith D et al (2016) Quality of end-of-life care provided to patients with different serious illnesses. JAMA Intern Med 176:1095-1102. https://doi.org/10.1001/ jamainternmed.2016.1200

29. Watson M, BrownE, ChambersEJ, Eggeling C (2007) End of life care in nephrology: from advanced disease to bereavement, 1. Aufl. Oxford University Press, Oxford, New York

30. Gerhard C (2012) Nephro-palliative care. https://www.staempfliverlag.com/detail/ISBN9783456850719. Zugegriffen: 11. Juli 2021

\section{Consensus Paper "Palliative renal care"}

Aspects of palliative medicine such as withholding and withdrawal of dialysis, initiating conservative therapy and cooperative end-of-life care have increasingly become part of standard renal care. The corresponding transfer of knowledge of palliative medicine principles has so far been lacking in training and further education. This consensus paper proposes structured curricular training for the kidney team based on principles of palliative care.

\section{Keywords}

Nephrology $\cdot$ Terminal care $\cdot$ Palliative medicine $\cdot$ Education $\cdot$ Consensus
MED UPDATE SEMINARE

Nephro Update 2022

15. Nephrologie-Update-Seminar

13.-14. Mai 2022

Berlin und Livestream

Wiss. Leitung:

Prof. Dr. Kai-Uwe Eckardt, Berlin

Prof. Dr. Jürgen Floege, Aachen

Unter der Schrimherrschaft der

DGfN, DN, GPN, DGIM, KfH

www.nephro-update.com

Auskunft für alle Update-Seminare: med update $\mathrm{GmbH}$

www.med-update.com

Tel.: 0611 - 736580

info@med-update.com 